\title{
ON THE OWEN SET OF TRANSPORTATION SITUATIONS
}

\begin{abstract}
This paper presents an axiomatic characterization of the Owen set of transportation games. In the characterization we use six properties including consistency (CONS2) and splitting and merging (SM) which are firstly proposed and defined for this setup in the present paper.
\end{abstract}

KEY WORDS: Transportation situations, cooperative games, Owen set

\section{INTRODUCTION}

In transportation situations, how to obtain the optimal solution for a single decision-maker is well known but a new problem arises when the agents involved cooperate and they have to distribute the obtained profit or saving among each other. In the cooperative game theory literature, we can find many general procedures (solution concepts) for distributing the total amount obtained by a set of agents among each other. In order to select the most suitable procedure of distribution or allocation, it is usual to characterize the solution concepts through different sets of properties. Nowadays, many operations research problems are studied from a game theoretical point of view introducing the very realistic multiple agents component in the analysis of such problems. For a survey on this interesting topic see Borm et al. (2002). In particular, transportation situations are studied from a game theoretical point of view in Samet et al. (1984), Sánchez-Soriano (1998) and Sánchez-Soriano et al. (2001) among others.

The purpose of this paper is to investigate certain aspects of transportation games and give an axiomatic characterization of its Owen set (a solution concept related to the dual optimal solutions of the transportation problem). To achieve this objective, we look at two operations research problems closely 
related to transportation situations: assignment situations and linear production situations. Assignment situations and linear production situations were studied from a game theoretical point of view in Shapley and Shubik (1972) and Owen (1975) respectively. A very interesting property in assignment games is that the core and the Owen set of the game coincide. This fact does not occur in linear production situations and transportation situations. Sasaki (1995) provided two characterizations of the core of assignment games and hence of its Owen set, and van Gellekom et al. (2000) provided a characterization of the Owen set of linear production games. Transportation situations occupy an intermediate position in between assignment and linear production situations because assignment situations are a special case of transportation situations and these can be rewritten as linear production situations. This particular position and relationship make us look into the properties used to characterize the Owen set of assignment games and linear production games to find the set of properties that characterize the Owen set of transportation games.

This paper consists of three sections. In the next section we present the most relevant definitions and results for the transportation situations and related games. We study the properties of the Owen set in Section 3. We consider two types of properties which are related to those introduced by Sasaki (1995) to characterize the core of assignment games, and by van Gellekom et al. (2000) to characterize the Owen set of linear production games. In the final section, we provide a characterization of the Owen set in transportation situations.

\section{TRANSPORTATION SITUATIONS AND GAMES}

A transportation situation is determined by a tuple $T=(P, Q, \mathbf{B}, \mathbf{p}, \mathbf{q})$, where $P$ and $Q$ are, respectively, the sets of supply points and demand points. The transport of one unit of the goods from supply point $i$ to demand point $j$ generates a non-negative profit of $b_{i j}$. The matrix $\mathbf{B}=\left[b_{i j}\right]_{i \in P, j \in Q}$ contains all the profits per unit of the goods. The supply at point $i \in P$ equals $p_{i}$ units of the goods and the demand $j \in Q$ is $q_{j}$ units 
where both $p_{i}$ and $q_{j}$ are non-negative integers. The vectors $\mathbf{p}=\left(p_{i}\right)_{i \in P}$ and $\mathbf{q}=\left(q_{j}\right)_{j \in Q}$ contain, respectively, the supplies and demands of the goods. The set of transportation situations is denoted by $G^{T}$.

A transportation plan for $T \in G^{T}$ is a matrix $\boldsymbol{\mu}=\left[\mu_{i j}\right]_{i \in P, j \in Q}$ where $\mu_{i j} \geq 0$ is the number of units of the goods that will be transported from supply point $i$ to demand point $j$. A supply point $i \in P$ can supply at most $p_{i}$ units of the goods, and a demand point $j \in Q$ wants to receive at most $q_{j}$. The maximal profit that can be obtained in this situation is

$$
\max \left\{\sum_{(i, j) \in P \times Q} b_{i j} \mu_{i j} \mid \boldsymbol{\mu} \text { is a transportation plan }\right\} \text {. }
$$

A transportation plan $\boldsymbol{\mu}$ is also called a feasible solution for the above transportation program $T$. We denote by $O_{p}(T)$ the set of optimal solutions for this program. Our interest is how to distribute the total profit among the agents when they cooperate, i.e., to propose a vector $(\mathbf{x} ; \mathbf{y}) \in \Re^{P} \times \Re^{Q}$ such that

$$
\sum_{i \in P} x_{i}+\sum_{j \in Q} y_{j}=\sum_{(i, j) \in P \times Q} b_{i j} \mu_{i j}^{*}
$$

where $\boldsymbol{\mu}^{*} \in O_{p}(T)$.

A well-known approach to distribute the total profit is to define a game for each transportation situation. Given $T \in G^{T}$, the corresponding transportation game $(N, w)$ is a cooperative transferable utility (TU) game with player set $N=P \cup Q$. Let $S \subset N, S \neq \varnothing$, be a coalition of players and define $P_{S}=P \cap S$ and $Q_{S}=Q \cap S$. If $S=P_{S}$ or $S=Q_{S}$, there are either only suppliers or demanders present, then no transport can take place and the worth $w(S)$ of coalition $S$ equals zero. Otherwise, the worth $w(S)$ depends upon the transportation plans for this coalition. A transportation plan $\boldsymbol{\mu}(S)$ for coalition $S$ is a transportation plan for the transportation problem $T_{S}=\left(P_{S}\right.$, $\left.Q_{S},\left[b_{i j}\right]_{i \in P_{S}, j \in Q_{S}},\left(p_{i}\right)_{i \in P_{S}},\left(q_{j}\right)_{j \in Q_{S}}\right)$. By convention $w(\varnothing)=0$.

Now, when thinking about how to share the profit among the suppliers and the demanders, one can consider sharing it 
according to some game theoretic solution concept for TUgames. For instance, one way is to do so according to an element in the core of the transportation game $(N, w)$, i.e.,

$C(w)=\left\{\begin{array}{l|l}(\mathbf{x} ; \mathbf{y}) \in \Re^{P} \times \Re^{Q} & \begin{array}{l}\sum_{i \in P} x_{i}+\sum_{j \in Q} y_{j}=w(N) \text { and } \\ \sum_{i \in P_{S}} x_{i}+\sum_{j \in Q_{S}} y_{j} \geq w(S), \quad \text { for all } S \subset N, S \neq \varnothing\end{array}\end{array}\right\}$.

If a core-element is proposed as a distribution of the total profit $w(N)$, then each coalition $S$ will get at least as much as it can obtain on its own, therefore no coalition has an incentive to split off. The transportation games have non-empty core and are superadditive but not convex. Thus, for an arbitrary transportation game we can always select a core element, but with great difficulty. Owen (1975) introduced the class of linear production games and presented a method to find a non-empty subset of the core of these games. Since a transportation game can be seen as a special case of linear production games, we can use this method to derive core elements. This set is the so-called $O$ wen set of the transportation situation, which is defined by

$$
\begin{aligned}
& \operatorname{Owen}(T)= \\
& \left\{\begin{array}{l}
(\mathbf{x} ; \mathbf{y}) \in \Re^{P} \times \Re^{Q} \mid \begin{array}{l}
x_{i}=p_{i} u_{i}, \forall i \in P, y_{j}=q_{j} v_{j}, \forall j \in Q, \\
\text { with }(\mathbf{u} ; \mathbf{v}) \in O_{d}(T)
\end{array}
\end{array}\right\},
\end{aligned}
$$

where $O_{d}(T)$ is the set of optimal solutions of the dual program of the (relaxed) transportation program for the grand coalition, i.e.,

$$
\min \left\{\sum_{i \in P} p_{i} u_{i}+\sum_{j \in Q} q_{j} v_{j} \mid u_{i}+v_{j} \geq b_{i j}, \quad u_{i}, v_{j} \geq 0, \text { for all } i \in P, j \in Q\right\} .
$$

Note that each element $u_{i}, i \in P, v_{j}, j \in Q$, of a vector $(\mathbf{u} ; \mathbf{v}) \in O_{d}(T)$ is the mean profit that an agent will obtain per unit from the supply or demand. Therefore, an element $\left(p_{i} u_{i}, q_{j} v_{j}\right)_{i \in P, j \in Q}$ of the Owen set is a vector of profits that players receive from the supply or demand.

Assignment situations are a special case of transportation situations when all supplies $p_{i}, i \in P$, and demands $q_{j}, j \in Q$, 
equal 1 . Hence, an assignment situation can be represented as $A=(P, Q, \mathbf{B})$. Assignment games were introduced by Shapley and Shubik (1972). They proved that the core of an assignment game is the non-empty set of optimal solutions of the dual program of the (relaxed) assignment program for the grand coalition, i.e., $C(w)=O_{d}(A)=\operatorname{Owen}(A)$. In the sequel, we will denote the set of assignment situations by $G^{A}$ and the core of the assignment game $C(w)$ by $C(A)$.

In general, the Owen set does not coincide with the core of a transportation game. See Sánchez-Soriano et al. (2001) for a detailed analysis of the relationship between the core and the Owen set of a transportation situation.

A solution concept $\Psi$ on $G^{T}$ is a map, which assigns to every $T=(P, Q, \mathbf{B}, \mathbf{p}, \mathbf{q}) \in G^{T}$ a non-empty set of available distributions $\varnothing \neq \Psi(T) \subset \Re^{P} \times \Re^{Q}$.

\section{PROPERTIES}

This section is devoted to the properties of the Owen set. We begin with those properties which are common to general games, or, at least to two-sided market games. We end up with the definition of two new properties which are related to those introduced by Sasaki (1995) to characterize the core of assignment games, and by van Gellekom et al. (2000) to characterize the Owen set of linear production games.

DEFINITION 1. A solution $\Psi$ on $G^{T}$ satisfies:

(EFF) Efficiency, if $x(P)+y(Q)=w^{T}(P \cup Q), \quad \forall(\mathbf{x} ; \mathbf{y}) \in$ $\Psi(T), \forall T \in G^{T}$.

(IR) Individual Rationality, if $(\mathbf{x} ; \mathbf{y}) \geq 0, \forall(\mathbf{x} ; \mathbf{y}) \in \Psi(T)$, $\forall T \in G^{T}$.

(CR) Couple Rationality, if $x_{i}+y_{j} \geq w^{T}(\{i, j\})=b_{i j} \min \left\{p_{i}\right.$, $\left.q_{j}\right\}, \forall i \in P, \forall j \in Q, \forall(\mathbf{x} ; \mathbf{y}) \in \Psi(T), \forall T \in G^{T}$.

(CONT) Continuity, if for every $(P, Q, \mathbf{B}, \mathbf{p}, \mathbf{q}) \in G^{T}$ and every sequence $\left\{T^{k}=\left(P, Q, \mathbf{B}^{k}, \mathbf{p}, \mathbf{q}\right)\right\}_{k \in \mathbb{N}}$ of transportation problems and elements $\left\{\left(\mathbf{x}^{\mathbf{k}} ; \mathbf{y}^{\mathbf{k}}\right) \in \Psi\left(T^{k}\right)\right\}_{k \in \mathbb{N}}$, such that: (a) $\mathbf{B}^{\mathbf{k}} \rightarrow \mathbf{B}$ (i.e. $b_{i j}^{k} \rightarrow b_{i j}$, for all $i \in P, j \in Q$ ); (b) $\left(\mathbf{x}^{\mathbf{k}} ; \mathbf{y}^{\mathbf{k}}\right) \rightarrow(\mathbf{x} ; \mathbf{y})$, it follows that $(\mathbf{x} ; \mathbf{y}) \in \Psi(T)$. 
PROPOSITION 1. The Owen Set satisfies (EFF), (IR), (CR) and (CONT) on $G^{T}$.

Proof. Since every allocation in the Owen set is a core element, it is obvious that the Owen set satisfies (EFF), (IR) and (CR). To prove the continuity of the Owen set, let $\left\{T^{k}=\right.$ $\left.\left(P, Q, \mathbf{B}^{\mathbf{k}}, \mathbf{p}, \mathbf{q}\right)\right\}_{k \in \mathbb{N}},\left\{\left(\mathbf{x}^{k} ; \mathbf{y}^{k}\right) \in \operatorname{Owen}\left(T^{k}\right)\right\}_{k \in \mathbb{N}}$, and let $T=(P$, $Q, \mathbf{B}, \mathbf{p}, \mathbf{q})$ and $(\mathbf{x} ; \mathbf{y})$, such that (i) $\mathbf{B}^{\mathbf{k}} \underset{k \rightarrow \infty}{\longrightarrow} \mathbf{B}$ and (ii) $\left(\mathbf{x}^{\mathbf{k}} ; \mathbf{y}^{\mathbf{k}}\right) \underset{k \rightarrow \infty}{\longrightarrow}(\mathbf{x} ; \mathbf{y})$.

$\left(\mathbf{x}^{\mathbf{k}} ; \mathbf{y}^{\mathbf{k}}\right) \in \operatorname{Owen}\left(T^{k}\right), \forall k \in \mathbf{N}$ then there exists $\left(\mathbf{u}^{\mathbf{k}} ; \mathbf{v}^{\mathbf{k}}\right) \in$ $O_{d}\left(T^{k}\right)$ such that

$$
x_{i}^{k}=p_{i} u_{i}^{k}, \forall i \in P \quad \text { and } \quad y_{j}^{k}=q_{j} v_{j}^{k}, \forall j \in Q, \forall k \in \mathbb{N} .
$$

Then, it follows from condition (ii) that

$$
u_{i}^{k}=\frac{x_{i}^{k}}{p_{i}} \underset{k \rightarrow \infty}{\longrightarrow} \frac{x_{i}}{p_{i}}, \quad \forall i \in P \quad \text { and } \quad v_{j}^{k}=\frac{y_{j}^{k}}{q_{j}} \underset{k \rightarrow \infty}{\longrightarrow} \frac{y_{j}}{q_{j}}, \quad \forall j \in Q .
$$

Let $(\mathbf{u} ; \mathbf{v})$ given by $u_{i}=x_{i} / p_{i}, \forall i \in P$, and $v_{j}=y_{j} / q_{j}, \forall j \in Q$. We will show that $(\mathbf{u} ; \mathbf{v}) \in O_{d}(T)$.

$\left(\mathbf{u}^{\mathbf{k}} ; \mathbf{v}^{\mathbf{k}}\right) \in O_{d}\left(T^{k}\right), \forall k \in \mathbb{N}$, then $\left(\mathbf{u}^{\mathbf{k}} ; \mathbf{v}^{\mathbf{k}}\right) \geq \mathbf{0}$, and $u_{i}^{k}+v_{j}^{k} \geq$ $b_{i j}^{k}, \forall i \in P, \forall j \in Q$. Therefore, taking (i) into account, it follows that $(\mathbf{u} ; \mathbf{v})=\lim _{k \rightarrow \infty}\left(\mathbf{u}^{\mathbf{k}} ; \mathbf{v}^{\mathbf{k}}\right) \in F_{d}(T)$, where $F_{d}(T)$ is the feasible set of the dual program of the (relaxed) transportation program for the grand coalition.

Now, in order to prove the optimality of $(\mathbf{u} ; \mathbf{v})$, we will find a feasible solution $\boldsymbol{\mu}$ for the transportation program $T$ (i.e. $\boldsymbol{\mu} \in F_{p}(T)$ ) with the same value for the objective function.

Since $\left(\mathbf{u}^{\mathbf{k}} ; \mathbf{v}^{\mathbf{k}}\right) \in O_{d}\left(T^{k}\right), \forall k \in \mathbb{N}$, then for all $k \in \mathbb{N}$, there exists $\boldsymbol{\mu}^{\mathbf{k}} \in O_{p}\left(T^{k}\right) \subset F_{p}\left(T^{k}\right)$ such that

$$
\sum_{i \in P} \sum_{j \in Q} b_{i j}^{k} \mu_{i j}^{k}=\sum_{i \in P} p_{i} u_{i}^{k}+\sum_{j \in Q} q_{j} v_{j}^{k}
$$

Note that the feasible set $F_{p}\left(T^{k}\right)=F_{p}(T)$, for all $k \in \mathbb{N}$, and $F_{p}(T)$ is compact. Then, the sequence $\left\{\boldsymbol{\mu}^{k}\right\}_{k \in \mathbb{N}} \subset F_{p}(T)$ has a convergent subsequence $\left\{\boldsymbol{\mu}^{k_{\lambda}}\right\}_{\lambda \in \mathbb{N}}$. Let $\boldsymbol{\mu}^{\mathbf{0}} \in F_{p}(T)$ its limit, then on the one hand 


$$
\lim _{\lambda \rightarrow \infty}\left(\sum_{i \in P} p_{i} u_{i}^{k_{\lambda}}+\sum_{j \in Q} q_{j} v_{j}^{k_{\lambda}}\right)=\lim _{\lambda \rightarrow \infty} \sum_{i \in P} \sum_{j \in Q} b_{i j}^{k_{\lambda}} \mu_{i j}^{k_{\lambda}}=\sum_{i \in P} \sum_{j \in Q} b_{i j} \mu_{i j}^{0} .
$$

On the other hand,

$$
\lim _{\lambda \rightarrow \infty}\left(\sum_{i \in P} p_{i} u_{i}^{k_{\lambda}}+\sum_{j \in Q} q_{j} v_{j}^{k_{\lambda}}\right)=\sum_{i \in P} p_{i} u_{i}+\sum_{j \in Q} q_{j} v_{j} .
$$

Thus, from (3) and (4) it follows that $(\mathbf{u} ; \mathbf{v}) \in O_{d}(T)$. Then, $(\mathbf{x} ; \mathbf{y}) \in \operatorname{Owen}(T)$.

The following property was introduced by Sasaki (1995) to characterize the core on assignment problems. Since the core coincides with the Owen set in the assignment problems, we will look at it in order to define a new consistency property for transportation situations. Then, we will use this new consistency to characterize the Owen set.

DEFINITION 2. A solution $\Phi$ on $G^{A}$ satisfies consistency (CONS), if for all $A=(P, Q, \mathbf{B}) \in G^{A}$, for all $(\mathbf{x} ; \mathbf{y}) \in \Phi(A)$ and for all $(\bar{P}, \bar{Q}) \subset(P, Q)$, such that there exists a feasible assignment $\boldsymbol{\mu} \in F_{p}(A)$ with

(i) $x(P)+y(Q)=\sum_{(i, j) \in P \times Q} b_{i j} \mu_{i j}$,

(ii) $\mu_{i j}=0$, for all pair $(i, j) \in(P \backslash \bar{P}) \times \bar{Q}$ or $(i, j) \in \bar{P} \times(Q \backslash \bar{Q})$,

(iii) $\sum_{i \in \bar{P}} x_{i}+\sum_{j \in \bar{Q}} y_{j}=\sum_{(i, j) \in \bar{P} \times \bar{Q}} b_{i j} \mu_{i j}$,

it follows that $\left(\left.\mathbf{x}\right|_{\bar{P}} ;\left.\mathbf{y}\right|_{\bar{Q}}\right) \in \Phi\left(\bar{P}, \bar{Q},\left.\mathbf{B}\right|_{\bar{P} \cup \bar{Q}}\right)$.

The following theorem can be found in Sasaki (1995).

THEOREM 1. Let $\Phi$ a solution on $G^{A}$ which satisfies (CONS) and (CONT). If $\Phi(A) \subset C(A)$, for all $A \in G^{A}$, then $\Phi \equiv C$ on $G^{A}$.

The Owen set satisfies (CONS) on $G^{A}$ (because it coincides with the core on that class), but not on $G^{T}$. Instead of (CONS) the Owen set satisfies on $G^{T}$ a slightly different version of the consistency property. 
DEFINITION 3. A solution $\Psi$ on $G^{T}$ satisfies (CONS2), if for all $T=(P, Q, \mathbf{B}, \mathbf{p}, \mathbf{q}) \in G^{T}$, for all $(\mathbf{x} ; \mathbf{y}) \in \Psi(T)$, and for all $(\bar{P}, \bar{Q}) \subset(P, Q)$, such that

$$
\sum_{i \in \bar{P}} x_{i}+\sum_{j \in \bar{Q}} y_{j}=w(\bar{P} \cup \bar{Q})
$$

it verifies that $\left(\left.\mathbf{x}\right|_{\bar{P}} ;\left.\mathbf{y}\right|_{\bar{Q}}\right) \in \Psi\left(\bar{P}, \bar{Q},\left.\mathbf{B}\right|_{\bar{P} \cup \bar{Q}},\left.\mathbf{p}\right|_{\bar{P}},\left.\mathbf{q}\right|_{\bar{Q}}\right)$.

PROPOSITION 2. The Owen set satisfies (CONS2) on $G^{T}$

Proof. Let $T=(P, Q, \mathbf{B}, \mathbf{p}, \mathbf{q}) \in G^{T}$, and let $(\mathbf{x} ; \mathbf{y}) \in \operatorname{Owen}(T)$ and $(\bar{P}, \bar{Q}) \subset(P, Q)$, satisfying the above condition.

Since $(\mathbf{x} ; \mathbf{y}) \in \operatorname{Owen}(T)$, there exists $(\mathbf{u} ; \mathbf{v}) \in O_{d}(T)$ such that $x_{i}=p_{i} u_{i}, \forall i \in P$ and $y_{j}=q_{j} v_{j}, \forall j \in Q$.

Clearly, $\left(\left.\mathbf{u}\right|_{\bar{P}} ;\left.\mathbf{v}\right|_{\bar{Q}}\right)$ belongs to $F_{d}\left(T_{\bar{P} \cup \bar{Q}}\right)$ and

$$
\sum_{i \in \bar{P}} p_{i} u_{i}+\sum_{j \in \bar{Q}} q_{j} v_{j}=\sum_{i \in \bar{P}} x_{i}+\sum_{j \in \bar{Q}} y_{j}=w(\bar{P} \cup \bar{Q}),
$$

so $\left(\left.\mathbf{u}\right|_{\bar{P}} ;\left.\mathbf{v}\right|_{\bar{Q}}\right) \in O_{d}\left(T_{\bar{P} \cup \bar{Q}}\right)$. Therefore, $\quad\left(\left.\mathbf{x}\right|_{\bar{P}} ;\left.\mathbf{y}\right|_{\bar{Q}}\right) \in \operatorname{Owen}(\bar{P}, \bar{Q}$, $\left.\left.\mathbf{B}\right|_{\bar{P} \cup \bar{Q}},\left.\mathbf{p}\right|_{\bar{P}},\left.\mathbf{q}\right|_{\bar{Q}}\right)$.

The following lemma will be useful in the axiomatic characterization of the Owen set.

LEMMA 1. Let $\Psi$ a solution on $G^{T}$ satisfying (EFF) and (CONS2). Then $\Psi$ satisfies (CONS) on $G^{A}$.

Proof. Let $A=(P, Q, \mathbf{B}) \in G^{A}, \quad(\mathbf{x} ; \mathbf{y}) \in \Psi(A) \quad$ and $(\bar{P}, \bar{Q}) \subset(P, Q)$ satisfying the conditions in Definition 2. By efficiency and condition (i), the matching $\boldsymbol{\mu}$ is an optimal assignment for $A$. And hence, by condition (ii), the matching $\left.\boldsymbol{\mu}\right|_{\bar{P} \times \bar{Q}}$ is an optimal assignment for $\left.A\right|_{\bar{P} \cup \bar{Q}}$. Therefore, by condition (iii), it is satisfied that

$$
\sum_{i \in \bar{P}} x_{i}+\sum_{j \in \bar{Q}} y_{j}=w(\bar{P} \cup \bar{Q}) .
$$

Since $\Psi$ satisfies (CONS2), it follows that $\left(\left.\mathbf{x}\right|_{\bar{P}} ;\left.\mathbf{y}\right|_{\bar{Q}}\right) \in \Psi(\bar{P}, \bar{Q}$, $\left.\left.\mathbf{B}\right|_{\bar{P} \cup \bar{Q}}\right)$. 
To each transportation situation $T=(P, Q, \mathbf{B}, \mathbf{p}, \mathbf{q}) \in G^{T}$ a representatives assignment situation $A^{T}=\left(P^{T}, Q^{T}, \mathbf{B}^{T}\right) \in G^{A}$ can be associated by splitting every supply point $i \in P$ into $p_{i}$ supplier representatives and every demand point $j \in Q$ into $q_{j}$ demander representatives. In this way, we will have a set of suppliers $P^{T}=\left\{(\right.$ ir $\left.), i \in P, 1 \leq r \leq p_{i}\right\}$ and another set $Q^{T}=$ $\left\{(j c), j \in Q, 1 \leq c \leq q_{j}\right\}$ of demanders. The profit generated by matching a supplier (ir) and a demander $(j c)$ is $b_{i r, j c}^{T}:=b_{i j}$. Clearly, the corresponding operations research problems in both situations are equivalent, as are the optimal solutions.

The following property adapts the property of shuffle (van Gellekom et al., 2000) to the context of transportation situations. Note that our property is much less restrictive. We restrict ourselves to integer divisions and unions and we do not ask for permutation invariance. In our context, the following property can be interpreted in terms of a non-manipulability condition.

DEFINITION 4. A solution $\Psi$ on $G^{T}$ satisfies Splitting and Merging (SM) if for every $T \in G^{T}$ the following conditions are satisfied:

(S) If $(\mathbf{x} ; \mathbf{y}) \in \Psi(T)$, then there exists $\left(\mathbf{x}^{A^{T}} ; \mathbf{y}^{A^{T}}\right) \in \Psi\left(A^{T}\right)$ such that

$$
x_{i}=\sum_{r=1}^{p_{i}} x_{i r}^{A^{T}}, \forall i \in P, \quad y_{j}=\sum_{c=1}^{q_{j}} y_{j c}^{A^{T}}, \forall j \in Q .
$$

(M) If $\left(\mathbf{x}^{A^{T}} ; \mathbf{y}^{A^{T}}\right) \in \Psi\left(A^{T}\right)$, then the distribution $(\mathbf{x} ; \mathbf{y}) \in \Psi(T)$, where

$$
x_{i}=\sum_{r=1}^{p_{i}} x_{i r}^{A^{T}}, \forall i \in P, \quad y_{j}=\sum_{c=1}^{q_{j}} y_{j c}^{A^{T}}, \forall j \in Q .
$$

PROPOSITION 3. The Owen Set satisfies (SM).

Proof. We begin by proving the splitting part. We outline the proof as follows. Let $T \in G^{T}$ and $(\mathbf{x} ; \mathbf{y}) \in \operatorname{Owen}(T)$, then there exists $(\mathbf{u} ; \mathbf{v}) \in O_{d}(T)$ such that $x_{i}=p_{i} u_{i}$, for all $i \in P$, and $y_{j}=q_{j} v_{j}, \forall j \in Q$.

Let $A^{T} \in G^{A}$ the assignment situation derived from $T$. It can easily be checked that $\left(\mathbf{u}^{\mathbf{A}} ; \mathbf{v}^{\mathbf{A}}\right) \in O_{d}\left(A^{T}\right)$, where 


$$
\begin{aligned}
& u_{i r}^{A}=u_{i}=\frac{x_{i}}{p_{i}}, \forall i \in P, 1 \leq r \leq p_{i}, \\
& v_{j c}^{A}=v_{j}=\frac{y_{j}}{q_{j}}, \forall j \in Q, \quad 1 \leq c \leq q_{j} .
\end{aligned}
$$

Then, the allocation $\left(\mathbf{x}^{A^{T}} ; \mathbf{y}^{A^{T}}\right)$ defined as

$$
\begin{aligned}
& x_{i r}^{A}=u_{i r}^{A}=\frac{x_{i}}{p_{i}}, \forall i \in P, 1 \leq r \leq p_{i}, \\
& y_{j c}^{A}=v_{j c}^{A}=\frac{y_{j}}{q_{j}}, \forall j \in Q, 1 \leq c \leq q_{j},
\end{aligned}
$$

belongs to Owen $\left(A^{T}\right)$, with $x_{i}=\sum_{r=1}^{p_{i}} x_{i r}^{A}$, for all $i \in P$, and $y_{j}=\sum_{c=1}^{q_{j}} y_{j c}^{A}$, for all $j \in Q$.

For the merging condition, let $\left(\mathbf{x}^{A^{T}} ; \mathbf{y}^{A^{T}}\right) \in \operatorname{Owen}\left(A^{T}\right)$. Notice that for an assignment situation, $A \in G^{A}$, Owen $(A)=$ $O_{d}(A)$. It can easily be checked that the vector $(\overline{\mathbf{u}} ; \overline{\mathbf{v}})$ of averages prices, given by

$$
\bar{u}_{i}=\frac{\sum_{r=1}^{p_{i}} x_{i r}^{A^{T}}}{p_{i}}, \forall i \in P, \quad \bar{v}_{j}=\frac{\sum_{c=1}^{q_{j}} y_{j c}^{A^{T}}}{q_{j}}, \forall j \in Q,
$$

belongs to $O_{d}(T)$. Therefore, the allocation $(\mathbf{x} ; \mathbf{y})$ given by $x_{i}=p_{i} \bar{u}_{i}, \forall i \in P$, and $y_{j}=q_{j} \bar{v}_{j}, \forall j \in Q$, belongs to $\operatorname{Owen}(T)$ and

$$
x_{i}=\sum_{r=1}^{p_{i}} x_{i r}^{A^{T}}, \forall i \in P, \quad y_{j}=\sum_{c=1}^{q_{j}} y_{j c}^{A^{T}}, \forall j \in Q .
$$

\section{AXIOMATIC CHARACTERIZATION}

Now, we offer an axiomatization involving the properties analyzed in the previous section. We will show that the set of axioms we have considered are logically independent.

THEOREM 2. There is a unique solution on $G^{T}$ that satisfies (EFF), (IR), (CONS2), (CR), (SM), and (CONT), and it is the Owen set.

Proof. Let $\Psi$ solution on $G^{T}$ that satisfies (EFF), (IR), (CONS2), (CR), (SM), and (CONT). 
First, we will prove that $\Psi \subset$ Owen $(\cdot)$. Let $T \in G^{T}$, and $(\mathbf{x} ; \mathbf{y}) \in \Psi(T)$. Since $\Psi$ satisfies (SM), then it follows from the splitting condition that

$$
x_{i}=\sum_{r=1}^{p_{i}} x_{i r}^{A^{T}}, \forall i \in P, y_{j}=\sum_{c=1}^{q_{j}} y_{j c}^{A^{T}}, \forall j \in Q,
$$

for some $\left(\mathbf{x}^{A^{T}} ; \mathbf{y}^{A^{T}}\right) \in \Psi\left(A^{T}\right)$, where $A^{T}$ is the assignment situation derived from the transportation situation $T$. By (IR), it follows that

$$
\begin{aligned}
& x_{i r}^{A^{T}} \geq v^{A^{T}}(\{i r\})=0, \quad \forall i \in P, \forall r=1, \ldots, p_{i}, \\
& y_{j c}^{A^{T}} \geq v^{A^{T}}(\{j c\})=0, \quad \forall j \in Q, \forall c=1, \ldots, q_{j} .
\end{aligned}
$$

On the other hand, because $\Psi$ satisfies (CR),

$$
\begin{aligned}
& x_{i r}^{A^{T}}+y_{j c}^{A^{T}} \geq w^{A^{T}}(\{i r, j c\})=b_{i r, j c}=b_{i j}, \\
& \quad \forall i \in P, \forall j \in Q, \quad 1 \leq r \leq p_{i}, \quad 1 \leq c \leq q_{j} .
\end{aligned}
$$

Then, it follows from (7) and (8) that $\left(\mathbf{x}^{A^{T}} ; \mathbf{y}^{A^{T}}\right) \in F_{d}\left(A^{T}\right)$. Moreover, since $\Psi$ satisfies $(\mathrm{EFF}), \quad\left(\mathbf{x}^{A^{T}} ; \mathbf{y}^{A^{T}}\right) \in O_{d}\left(A^{T}\right)=$ $\operatorname{Owen}\left(A^{T}\right)$.

Since Owen $(\cdot)$ satisfies (SM), then by the merging condition it is verified that $(\mathbf{x} ; \mathbf{y})$, with

$$
x_{i}=\sum_{r=1}^{p_{i}} x_{i r}^{A^{T}}, \forall i \in P, \quad y_{j}=\sum_{c=1}^{q_{j}} y_{j c}^{A^{T}}, \forall j \in Q,
$$

belongs to $\operatorname{Owen}(T)$.

Next, we will prove that Owen $(\cdot) \subset \Psi$. We have just proved that every solution satisfying the above properties is a subsolution of the Owen set. Because the Owen set coincides with the core on $G^{A}$, then $\Psi(A) \subset C(A)$, for all $A \in G^{A}$. Moreover, by Lemma $1, \Psi$ satisfies (CONS) on $G^{A}$, and by hypotheses, $\Psi$ also satisfies (CONT). Therefore, by Theorem 1, $\Psi(A)=C(A)=$ Owen $(A)$, for all $A \in G^{A}$.

Let $(\mathbf{x} ; \mathbf{y}) \in \operatorname{Owen}(T)$, which satisfies (SM). Then, it follows from the merging condition that there exist $\left(\mathbf{x}^{A^{T}} ; \mathbf{y}^{A^{T}}\right) \in$ Owen $\left(A^{T}\right)$ verifying (5). 
Since $\Psi\left(A^{T}\right)=$ Owen $\left(A^{T}\right)$, it follows that $\left(\mathbf{x}^{A^{T}} ; \mathbf{y}^{A^{T}}\right) \in$ $\Psi\left(A^{T}\right)$. Thus, taking that $\Psi$ satisfies the merging condition into account, it is verified that $(\mathbf{x} ; \mathbf{y}) \in \Psi(T)$.

PROPOSITION 4. The axioms (EFF), (IR), (CR), (CONS2), (SM) and (CONT) are logically independent.

Proof. To show the independence of these six axioms we will prove that for every group of five axioms there exists a solution which satisfies them all except the sixth one.

$(\neg \mathrm{EFF})$ Let $\Psi^{1}(T)=\left\{(\mathbf{x} ; \mathbf{y})=\left(p_{i} u_{i}, q_{j} v_{j}\right)_{i \in P, j \in Q} \mid(\mathbf{u} ; \mathbf{v}) \in F_{d}(T)\right\}$, for all transportation situation $T \in G^{T}$. Then, the solution concept $\Psi^{1}$ trivially satisfies all axioms except efficiency.

Note that every distribution $(\mathbf{x} ; \mathbf{y})=\left(p_{i} u_{i}, q_{j} v_{j}\right)_{i \in P, j \in Q}$ with $u_{i}+v_{j} \geq b_{i j}$, for all $(i, j) \in P \times Q$ satisfies couple rationality. In fact,

$$
\begin{aligned}
& x_{i}+y_{j} \geq p_{i} u_{i}+q_{j} v_{j} \geq \min \left\{p_{i}, q_{j}\right\} b_{i j}=w(\{i, j\}), \\
& \quad \text { for all } i \in P, j \in Q .
\end{aligned}
$$

The splitting and merging property is trivially accomplished because the distributions in $\Psi^{1}$ only relay on the linear programming program of the underlying transportation problem, which is invariant under the operations of merging and splitting. With respect to the consistency property, the restriction to a coalition $S \subset N=P \cup Q$ of a feasible solution for the dual program of the (relaxed) transportation program for the grand coalition is also feasible for the corresponding dual program of the (relaxed) transportation program for coalition $S$.

The proof for continuity resembles that of proposition 1 to a high degree.

( $\neg$ IR)The solution concept on $G^{T}, \Psi^{2}$, defined as

$$
\Psi^{2}(T)=\left\{\begin{array}{l|l}
(\mathbf{x} ; \mathbf{y}) \in \Re^{P \cup Q} \mid \begin{array}{l}
\sum_{i \in P} x_{i}+\sum_{j \in Q} y_{j}=w(N), x_{i}=p_{i} u_{i}, y_{j}=q_{j} v_{j}, \forall(i, j) \in P \times Q, \\
\operatorname{with}(\mathbf{u} ; \mathbf{v}) \in \Re^{P \cup Q} \text { s.t. } u_{i}+v_{j} \geq b_{i j}, \forall(i, j) \in P \times Q
\end{array}
\end{array}\right\},
$$

satisfies all axioms except individual rationality. Note that the non-negativity condition of the dual program has been ignored, whereas the efficiency condition and couple rationality have been imposed. With respect to consistency, continuity and 
splitting and merging, the argument is similar to that $\Psi^{1}$ considered above, taking into account that efficiency for coalition $S$ is a necessary condition to ask for consistency.

( $\neg \mathrm{CR}$ ) The solution concept on $G^{T}, \Psi^{3}$, defined as

$$
\Psi^{3}(T)=\left\{\begin{array}{l|l}
(\mathbf{x} ; \mathbf{y}) \in \Re^{P \cup Q} & \begin{array}{l}
\sum_{i \in P} x_{i}+\sum_{j \in Q} y_{j}=w(N), \quad x_{i}=p_{i} u_{i}, \\
y_{j}=q_{j} v_{j}, u_{i} \geq 0, v_{j} \geq 0, \forall(i, j) \in P \times Q
\end{array}
\end{array}\right\},
$$

satisfies all axioms except couple rationality, since the inequalities $u_{i}+v_{j} \geq b_{i j}, i \in P, j \in Q$ of the dual program have been ignored, whereas the efficiency condition and individual rationality have been imposed. With respect to consistency, continuity and splitting and merging, the argument is similar to that of $\Psi^{2}$ considered above.

( ᄀCONS2) The solution concept on $G^{T}, \Psi^{4}$, defined as

$$
\Psi^{4}(T)= \begin{cases}\left(p_{1} b_{11}, 0\right), & \text { if }|P|=|Q|=1 \text { and } p_{1}=q_{1} \\ \text { Owen }(T), & \text { otherwise }\end{cases}
$$

satisfies all axioms except consistency.

Let us consider the transportation problem $T$ with two suppliers and two demanders, supply vector $\mathbf{p}=(1,2)$, demand vector $\mathbf{q}=(1,1)$ and transportation matrix $\mathbf{B}=\left(\begin{array}{cc}10 & 5 \\ 5 & 0\end{array}\right)$. The distribution $(\mathbf{x} ; \mathbf{y})=(5,0 ; 5,0) \in \Psi^{4}(T)$ and is efficient for coalition $S=\{1,3\}$, but $\left(\mathbf{x}^{S} ; \mathbf{y}^{S}\right)=(5 ; 5) \notin \Psi^{4}\left(T_{S}\right)=\{(10 ; 0)\}$.

$(\neg \mathrm{SM})$ If we remove the splitting and merging property then the core satisfies the remaining properties. We refer to SánchezSoriano (1998) for a proof of the continuity.

$(\neg C O N T)$ If we eliminate continuity a solution concept which chooses the relative interior of the Owen set invalidates the result.

\section{ACKNOWLEDGMENTS}

This research is supported by Oficina de Ciència i Tecnologia de la Generalitat Valenciana, through project GV-CTIDIA2002-32 and by Government of Spain, through a joint research 
grant Universidad Miguel Hernández-Università degli Studi di Genova HI2002-0032.

\section{REFERENCES}

Borm, P., Hamers, H. and Hendrickx, R. (2001), Operations research games: A survey, TOP 9, 139-216.

Owen, G. (1975), On the core of linear production games, Mathematical Programming 9, 358-370.

Samet, D., Tauman, Y. and Zang, I. (1984), An application of the AumannShapley prices for cost allocation in transportation problems, Mathematics of Operations Research 9, 25-42.

Sánchez-Soriano, J. (1998), El problema del transporte. Una aproximación desde la Teoría de Juegos (in Spanish). Ph.D. Thesis, University of Murcia, Murcia, Spain.

Sánchez-Soriano, J., López, M.A. and García-Jurado, I. (2001), On the core of transportation games, Mathematical Social Sciences 41, 215-225.

Sasaki, H. (1995), Consistency and monotonicity in assignment problems, International Journal of Game Theory 24, 373-397.

Shapley, L.S. and Shubik, S. (1972), The assignment game I: The core, International Journal of Game Theory 1, 111-130.

van Gellekom, J.R.G., Potters, J.A.M., Reijnierse, J.H., Engel M.C. and Tijs, S.H. (2000), Characterization of the Owen set of linear production processes, Games and Economic Behavior 32, 139-156.

Address for correspondence: Natividad Llorca, Dpto. Estadística y Matemática Aplicada and C.I.O., Universidad Miguel Hernández de Elche Edificio Torretamarit. Elche, 03202, Spain. E-mail: nllorca@umh.es

Elisenda Molina, Manuel A. Pulido, Joaquín Sánchez-Soriano, Dpto. Estadística y Matemática Aplicada and C.I.O., Universidad Miguel Hernández de Elche, Elche, 03202, Spain E-mail: \{e.molina, manpul,joaquin\}@,umh.es 
Reproduced with permission of the copyright owner. Further reproduction prohibited without permission. 